\title{
Automated unified system for LPG using microcontroller and GSM module- A Review
}

\author{
B. B. Didpaye ${ }^{1}$, Prof. S. K. Nanda ${ }^{2}$ \\ Student of M.E., Department of Electronics \& Telecommunication Engineering, P. R. Patil College of Engineering, \\ Amravati Maharashtra, India ${ }^{1}$ \\ HOD, Department of Electronics \& Telecommunication Engineering, P. R. Patil College of Engineering, Amravati \\ Maharashtra, India ${ }^{2}$
}

\begin{abstract}
A cost-effective, Automated Unified System for Liquefied Petroleum Gas (LPG) booking, leakage detection, real time gas monitoring system and automatic controlling of LPG regulator is proposed in this paper. The aim of this paper is to monitor for LPG leakage to avoid fire accidents providing house safety feature where security has been an important issue. The system detects the leakage of the LPG using gas sensor MQ6 and alerts the consumer about the gas leakage by sending SMS using GSM module and simultaneously activating the alarm and exhaust fan. The system additionally provides the automatically controlling of LPG regulator. To avoid the blast this system will disconnect the main power supply using relay. The additional advantage of the system is that it continuously monitors the level of the LPG present in the cylinder using weight sensor and automatically books the cylinder using a GSM module.
\end{abstract}

Keywords: LPG, gas cylinder monitoring, gas leakage detection and prevention, GSM, Alarm

\section{INTRODUCTION}

Liquefied Petroleum Gas commonly known as LPG consists of mixture of Commercial Propane and Commercial Butane having saturated as well as unsaturated hydrocarbons. It is odourless gas due to which Ethanethoil is added as powerful odorant, so that leakage can be easily detected. LPG is commonly used in homes for central heating, hot-water, gas fires, cooking and in mobile heaters for leisure activities such as boats, caravans and barbecues .This energy source is primarily composed of propane and butane which is highly flammable chemical compounds. LPG first produced in 1910 by Dr. Walter Snelling. It is classified as hazardous material because of its flammable properties and explosive potential when stored under pressure. Before the development of electronic household gas detectors in the 1980 s and 90s, gas presence was detected with a chemically infused paper that changed its colour when exposed to the gas. Since then, many technologies and devices have been developed to detect, monitor, and alert the leakage of a wide array of gases. Hence the requirement of an efficient system to measure and display the level of LPG is inevitable, which may be used for domestic and commercial purposes. Here we intend to propose a microcontroller based system where a gas sensor, MQ6 is used to detect dangerous gas leaks. This unit is incorporated into an alarm unit, to sound an alarm or give a visual indication of the LPG leakage. The sensor has good sensitivity combined with a quick response time at low cost. If leakage is detected, message to the authorized person or family member using cellular network called GSM is sent automatically. It also provides a feature to measure weight LPG cylinder with its value on LCD display. A gas quantity of less or equal to $7 \mathrm{~kg}$ books the cylinder automatically by sending text message to a dealer. Also when cylinder weighs less than or equal to 0.5
$\mathrm{Kg}$, it informs the family members by sending a message to refill the cylinder.

\section{LITERATURE SURVEY}

[1]A cost-effective, automatic Liquefied Petroleum Gas (LPG) booking, leakage detection and real time gas monitoring system is proposed in this paper. In this system, the LPG leakage is detected through the sensor and information is sent to the user by Short Message Service (SMS) and simultaneously alerts the customer using a GSM module, while activating the alarm and exhaust fan. The additional advantage of the system is that it continuously monitors the level of the LPG present in the cylinder using weight sensor and automatically books the cylinder using a GSM module.

[2] The system detects the leakage of the LPG and alerts the consumer about the leak and as an emergency measure the system will switch on the exhaust fan and also checks the leakage. An added feature of the system is that the approximate consumption is indicated in terms of the total weight. The proposed system makes use of GSM module in order to alert about the gas leakage via an SMS. Whenever the system detects the increase in the concentration of the LPG it immediately alerts by activating an alarm and simultaneously sending message to the specified mobile phones. The exhaust fan is switched on and an LPG safe solenoid valve fitted to the cylinder is given a signal to close avoiding further leakage. The device ensures safety and prevents suffocation and explosion due to gas leakage.

[3]The former systems can not react in time, even cannot obtain data from an accident and locate accurately. This system gives real time detective of potential risk area, collect the data of leak accident and locate leakage point. This system having protection circuitry consists of exhaust 
fan and a Liquefied Petroleum Gas Safe Solenoid Valve. The hazardous gasses like Liquefied Petroleum Gas and Propane were sensed and displayed each and every second in Liquid Crystal Display. If these gasses exceed normal level then alarm is generated immediately. In this system MQ-6 gas sensor used to sense poisonous gas and has high sensitivity to LPG and also response to natural gas. This work modifies the existing safety model installed in industries. It offers quick response time and accurate detection.

[4]Safety plays a major role in today's world and it is necessary that good safety systems are to be implemented in places of education and work. This work modifies the existing safety model installed in industries and this system also be used in homes and offices. The main objective of the work is design in microcontroller based toxic gas detecting and alerting system. The hazardous gases like LPG and propane were sensed and displayed each and every second in the LCD display. If these gases exceed the normal level then an alarm is generated immediately and also an alert message (SMS) is sent to the authorized person through the GSM. The advantage of this automated detection and alerting system over the manual method is that it offers quick response time and accurate detection of an emergency and in turn leading faster diffusion of the critical situation.

[5]The aim of this project is to monitor for liquid petroleum gas (LPG) leakage to avoid fire accidents providing house safety feature where security has been an important issue. The system detects the leakage of the LPG using gas sensor and alerts the consumer about the gas leakage by sending SMS. The proposed system uses the GSM to alert the person about the gas leakage via SMS. When the system detects the LPG concentration in the air exceeds the certain level then it immediately alert the consumer by sending SMS to specified mobile phone and alert the people at home by activating the alarm which includes the LED, Buzzer simultaneously and display the message on LCD display to take the necessary action and switch on the exhaust fan to decrease the gas concentration in the air.

[6]Gas leakage is a major concern with residential, commercial premises and gas powered transportation vehicles. One of the preventive measures to avoid the danger associated with gas leakage is to install a gas leakage detector at vulnerable locations. The objective of this work is to present the design of a cost effective automatic alarming system, which can detect liquefied petroleum gas leakage in various premises. Test results are demonstrated for an USB powered gas leakage detection system and it gives early warning signals under less severe conditions and activates a high pitched alarm in case of emergency situations to safeguard the users.

\section{PROPOSED METHODOLOGY}

This paper proposes an advance and innovative approach for LPG leakage detection, prevention and automatic booking for refill. In advance, the system provides the automatic controlling of LPG regulator also if leakage is detected the system will automatically turn off the main switch of power supply. Hence it helps to avoid the explosion and blast.

\section{A. System Architecture}

System block diagram comprises of following parts shown in figure 1. It consists of microcontroller (ATMega16A), gas sensor (MQ6), weight sensor (Load Cell-L6D), GSM module (SIMCOM 300), and display(s). Microcontroller ATMega 16 is the base of the system. The inputs given to the ATMega 16 are the output of gas sensor MQ-6, contactor and load cell L6D .The output of ATMega 16 are given to the SIMCOM 300 and LCD $16 \times 2$ display.

1) Microcontroller:

A very efficient and fast working controller is needed to continuously sense the LPG gas and its level (weight) sensor's output. Also a fast reply is desired when leakage is found. Along with this a system must possess capacity to store some information which can be used for further processing. Hence above operations require a very fast, single cycle execution rate microcontroller like ATMega16A. As shown in above figure 1, the microcontroller is at the centre of the system. It is having features like $16 \mathrm{~Kb}$ internal RAM making easy storage of entire code in microcontroller itself, also the 1 MIPS per $\mathrm{MHz}$ instruction cycle execution rate enhanced overall system performance. The LCD module connected to port B of ATMega16A in 4-bit mode is used to display the required messages. GSM module using AT commands connected to Rx and Tx pins of port D of ATMega16A are used to receive and transmit messages to desired family members and distributor. The weight sensor module output taken from relay circuit is connected to pins of port A which is used to continuously monitor gas level. The contactor is connected to the port C of ATMega16A is used to controlling the main power supply switch in the home.

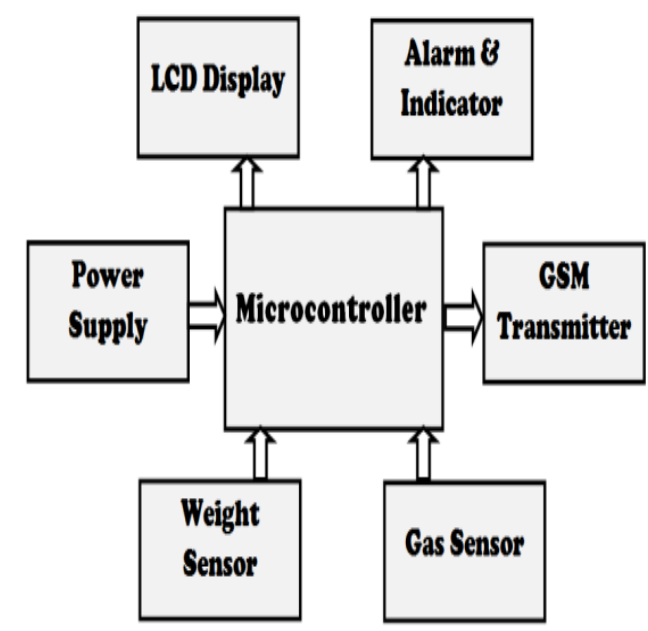

Fig. 1. System block diagram

2) Gas Sensor:

MQ6 is a semiconductor type gas sensor which detects the gas leakage. The sensitive material of MQ-6 is tin dioxide ( $\mathrm{SnO} 2)$. It has very low conductivity in clean air and its 
sensitivity increases with the concentration of gas, also it avoids gases like cooking fumes. It requires a voltage of 0 5 volts which is low and safe as per as the gaseous environment is considered. This sensor continuously senses the gas, and if concentration level goes above danger level then it turns $\mathrm{ON}$ relay which gives interrupt to microcontroller and alternately switches on buzzer and exhaust fan. This Gas sensor not only has sensitivity to propane and butane but also to other natural gases, low sensitivity to cigarette smoke an alcohol. This sensor can also be used for detection of other combustible gas such methane.

\section{3) Weight Sensor:}

For booking of refill from a distributor, we must be aware in advance of amount of gas in the cylinder, and for this purpose the level of gas present in the cylinder has to be monitored continuously. The load cell having required weighing capacity for domestic cylinder is used and for calibration purpose the weight sensor module is used along with the load cell. L6D weight sensor module is implemented in the system. The load cell output drives a relay circuit which gives two logic pulses (for $<=7 \mathrm{~kg}$ and $<=0.5 \mathrm{~kg}$ ) which are further connected to microcontroller port pins to detect the gas level.

4) GSM Module:

Gas sensor detects the presence of gas, weight sensor gives the gas level in cylinder, and microcontroller will take corrective or necessary actions. The status of all these happening has to be conveyed to the owner of system or housemates. GSM module is used to send an SMS to the user cell phone. When the gas leakage is detected by the gas sensor, microcontroller sends a signal to GSM module, in which one of the tasks is to send the text SMS. GSM module requires one SIM card. This module is capable to accept any network SIM card. The GSM module used is SIMCOM 300 which uses SIM memory to store the number of system owner or housemates and distributor or to whoever the messages have to be forwarded. It requires very less memory to send and receive text messages and operates on simple 12 Volt

5) Displays:

As the system performs controlling and monitoring operations, it is primary requirement to put a display in the system which shows various message such as gas leakage detection, booking number of cylinder in case of refill of cylinder and also will display actions taken by microcontroller. The system requires two Liquid Crystal Display (LCD) one of $16 \mathrm{X} 2$ characters operating on +5 Volt supply and operated in 4-bit mode is implemented for the task of displaying required messages. And another is seven segment display to display monitored weight of LPG. Interfacing with ATMega16A and short code of programming makes it very useful to make system more users friendly.

\section{B. Gas Leakage Detection and Prevention}

The system mainly consists of LPG leakage detection system, Microcontroller with GSM module and protection circuitry. The main function of gas leakage detection module which consist of gas sensor to continuously detect the gas leakage in the air. For the gas leakage detection
MQ6 placed in the vicinity of the gas cylinder. In the advent of leakage, the resistance of the sensor decreases increasing its conductivity. Corresponding pulse is fed to microcontroller and results in simultaneously switches on the buzzer and exhaust fan which we can reset by a manual reset switch. MQ6 gas sensor which offers many advantages like long lifetime, low cast, reliable and high sensitive to LPG. Also a logic high pulse $(+5 \mathrm{~V})$ is given as an interrupt to INT0 pin of ATMega16 Microcontroller. Microcontroller sends a message "EMERGENCY ALERT: LPG gas leakage found" to required cell numbers via GSM module and the same will be displayed on LCD. And with the help of Contactor the main power supply switch in home will turned OFF. Also another sensor is used to identify the requirement of LPG regulator ON or OFF. Hence a logic high pulse is given to INT1 pin of ATMega16 Microcontroller. It identifies the requirement and motor get started hence it turned ON the LPG regulator. In gas sensors Tin dioxide is the most common material, when any specified gases leak in the air, the electrical resistance in the sensor decreases. MQ6 gas sensor has less sensitivity to air but high sensitivity to combustible gases.

C. Automatic Gas Booking

In automatic Gas booking system, weight sensor L6D continuously monitors the weight of the gas in cylinder and displays it on seven segments LCD. When the weight of the gas is $<=7 \mathrm{Kg}$, a logic high pulse is fed to a port pin of microcontroller. As this pin goes high, microcontroller will send a booking message to gas agency of format, "REG_AMANGAS_12345". At the same time, the message will be displayed on LCD as "Booking Cylinder". When the weight of the gas goes below $1 \mathrm{~kg}$ another logic high pulse is fed to another port of microcontroller through a relay circuit as discussed in truth table (figure 2). As this port pin goes high, microcontroller will send a message as "Less LPG, Please Refill your Cylinder" through a GSM module to the customer's cell number and the message "Cylinder Empty, Please Refill" is displayed on the LCD display.

The output of gas sensor MQ-6, contactor and load cell L6D are given to the microcontroller. The output of ATMega 16 is given to the SIMCOM 300, regulator motor and LCD 16×2 display. The gas output of MQ6 is given to the INT0 pin of ATMega 16 as far as the highest priority is given to the leakage detection. The output of L6D is amplified and digitized by $\mathrm{A} / \mathrm{D}$ converter and is given to the port pins PA0 and PA1 of ATMega 16 as per the truth table figure 2 .

\begin{tabular}{|c|c|c|}
\hline PA0 & PA1 & Condition \\
\hline 0 & 0 & Full Cylinder \\
\hline 1 & 0 & Booking Cylinder $(<=7 \mathrm{~kg})$ \\
\hline 1 & 1 & Empty Cylinder $(<=0.5 \mathrm{~kg})$ \\
\hline \multicolumn{2}{r}{ Fig. 2. Truth Table }
\end{tabular}

\section{CONCLUSION}

Safety is a major concern in today's life. Currently there are so many approaches available for LPG leakage 
detection and automatic booking. To extract the features we will advance the system by using the new approach to increase the performance of system. Here in this paper we have given a new approach using contactor and regulator motor which will not only be simpler but also more efficient when applied.

\section{ACKNOWLEDGEMENT}

I would like to express my sincere gratitude to Prof. S. K. Nanda for the necessary support and guidance during various stages of work. I would also like to thank our management for the support because of which we are able to complete our research paper.

\section{REFERENCES}

11] B.D.Jolhe, P.A. Potdukhe, N.S.Gawai, "Automatic LPG Booking, Leakage Detection and real Time Gas Measurment Monitoring System", International Journal of Engineering Research and Technology (IJERT) Vol. 2, Issue 4, April 2013

[2] Sunithaa.J, Sushmitha.D, "Embedded control system for LPG leakage detection and prevention" International Conference on Computing and Control Engineering (ICCCE 2012), 12 \& 13 April, 2012

[3] Mr. Sagar Shinde, Mr .S. B. Patil, Dr. A. J. Patil, "Development of movable gas tanker leakage detection using wireless sensor network based on embedded system",International Journal of Engineering Research and Applications (IJERA) ISSN: 2248-9622 www.ijera.com Vol. 2, Issue 6, November- December 2012, pp.1180-1183

[4] V. Ramya, B. Palaniappan,"Embedded system for hazardous gas detection and alerting" International Journal of Distributed and Parallel Systems (IJDPS) Vol.3, No.3, May 2012

[5] S. Rajitha, T .Swapna, "A Security Alert System Using GSM for Gas Leakage", International Journal of VLSI and Embedded System (IJVES) Vol. 3, Issue 4, September-October 2012

[6] A. Mahalingam, R. T. Naayagi, N. E. Mastorakis, "Design and Implementation of an Economic Gas Leakage Detector", Recent Researches in Applications of Electrical and Computer Engineering

[7] ATMega 16 Datasheet; www.atmel.com

[8] Kelvin R. Sullivan, "Understanding Relays", A tutorial on relays.

[9] SIMCOM Ltd, 27th Dec, 2005, "SIM 300 Hardware Specification Manual"

[10] Display Elektronik GmbH Datasheet; LCD Module,DEM 16216 SYH-PY

[11] Technical Data MQ6 Gas Sensors, www.hwsensors.com

[12] Aluminum Single-Point Load Cell Datasheet, model 1004 www.vpgtransducers.co

\section{BIOGRAPHIES}

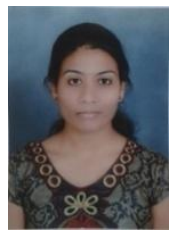

Ms. Bhagyashree B. Didpaye received B.E. degree in Electronics and TeleCommunication Engineering from Jawaharlal Darda Institute of Engineering and Technology Yavatmal in 2013 and currently she is pursuing M.E. (ExTC) in P .R. Patil College of Engineering, Amravati, Maharashtra, India. Her area of interest is Embedded System.

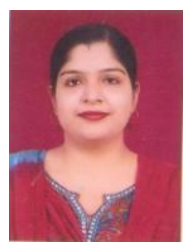

Prof. S. K. Nanda received M. E. degree and is now HOD, Department of Electronics and Telecommunication in $\mathrm{P}$. R. Patil College of Engineering, Amravati, Maharashtra, India. Her area of interest is Digital Electronics and has published 4 international and 2 national research papers till now. 